\title{
COEFFICIENT ESTIMATES FOR SAKAGUCHI TYPE FUNCTIONS
}

\author{
HALIT ORHAN, NIHAT YAGMUR AND MURAT ÇAĞLAR
}

\begin{abstract}
Let $S_{\lambda, \mu}^{n}(\alpha, t)$ be the class of normalized analytic functions defined in the open unit disk satisfying$$
\Re\left(\frac{(1-t) z\left(D_{\lambda, \mu}^{n} f(z)\right)^{\prime}}{D_{\lambda, \mu}^{n} f(z)-D_{\lambda, \mu}^{n} f(t z)}\right)>\alpha, \quad|t| \leq 1, t \neq 1
$$

for some $\alpha(0 \leq \alpha<1)$ and $D_{\lambda, \mu}^{n}$ is a linear multiplier differential operator defined by the authors in [2]. The object of the present paper is to discuss some properties of functions $f(z)$ belonging to the classes $S_{\lambda, \mu}^{n}(\alpha, t)$ and $T_{\lambda, \mu}^{n}(\alpha, t)$ where $f(z) \in T_{\lambda, \mu}^{n}(\alpha, t)$ if and only if $z f^{\prime}(z) \in S_{\lambda, \mu}^{n}(\alpha, t)$.
\end{abstract}

\section{INTRODUCTION}

Let $\mathcal{A}$ denote the family of functions $f$ of the form

$$
f(z)=z+\sum_{k=2}^{\infty} a_{k} z^{k}
$$

which are analytic in the open unit $\operatorname{disk} \mathcal{U}=\{z:|z|<1\}$. For $f(z)$ belongs to $\mathcal{A}$, the multiplier differential operator $D_{\lambda, \mu}^{n} f$ was defined by the authors in $[2]$ as follows

$$
\begin{aligned}
& D_{\lambda, \mu}^{0} f(z)=f(z) \\
& D_{\lambda, \mu}^{1} f(z)=D_{\lambda, \mu} f(z)=\lambda \mu z^{2}(f(z))^{\prime \prime}+(\lambda-\mu) z(f(z))^{\prime}+(1-\lambda+\mu) f(z) \\
& D_{\lambda, \mu}^{2} f(z)=D_{\lambda, \mu}\left(D_{\lambda, \mu}^{1} f(z)\right) \\
& D_{\lambda, \mu}^{n} f(z)=D_{\lambda, \mu}\left(D_{\lambda, \mu}^{n-1} f(z)\right)
\end{aligned}
$$

2000 Mathematics Subject Classification. 30C45.

Key words and phrases. Analytic function, coefficient estimates, Sakaguchi function, linear multiplier differential operator. 
where $\lambda \geqslant \mu \geqslant 0$ and $n \in \mathbb{N}_{0}=\mathbb{N} \cup\{0\}$.

If $f$ is given by (1.1) then from the definition of the operator $D_{\lambda, \mu}^{n} f(z)$ it is easy to see that

$$
D_{\lambda, \mu}^{n} f(z)=z+\sum_{k=2}^{\infty}[1+(\lambda \mu k+\lambda-\mu)(k-1)]^{n} a_{k} z^{k} .
$$

It should be remarked that the $D_{\lambda, \mu}^{n}$ is a generalization of many other linear operators considered earlier by different authors. In particular, for $f \in \mathcal{A}$ we have the following:

- $D_{1,0}^{n} f(z) \equiv D^{n} f(z)$ the operator investigated by Sălăgean (see [4]).

- $D_{\lambda, 0}^{n} f(z) \equiv D_{\lambda}^{n} f(z)$ the operator studied by Al-Oboudi (see [3]).

- $D_{\lambda, \mu}^{n} f(z)$ the operator firstly considered for $0 \leqslant \mu \leqslant \lambda \leqslant 1$, by Răducanu and Orhan (see [1]).

A function $f(z) \in \mathcal{A}$ is said to be in the class $S_{\lambda, \mu}^{n}(\alpha, t)$ if it satisfies

$$
\Re\left(\frac{(1-t) z\left(D_{\lambda, \mu}^{n} f(z)\right)^{\prime}}{D_{\lambda, \mu}^{n} f(z)-D_{\lambda, \mu}^{n} f(t z)}\right)>\alpha, \quad|t| \leq 1, t \neq 1
$$

for all $z \in \mathcal{U}$ and some $\alpha(0 \leq \alpha<1)$.

We also denote by $T_{\lambda, \mu}^{n}(\alpha, t)$ the subclass of $\mathcal{A}$ consisting of all functions $f(z)$ such that $z f^{\prime}(z) \in S_{\lambda, \mu}^{n}(\alpha, t)$. The class $S_{\lambda, \mu}^{0}(0,-1)$ was introduced by Sakaguchi [5]. Therefore, a function $f(z) \in S_{\lambda, \mu}^{0}(\alpha,-1)$ is called Sakaguchi function of order $\alpha$ (see [6] and [8] ). Further, the class $S_{\lambda, \mu}^{0}(\alpha, t)$ was introduced and studied by Owa et al. [7]. Various Sakaguchi type functions were investigated and studied by many authors including ([9], [10], [11]). We note that $S_{\lambda, \mu}^{0}(0,-1)$ is the class of starlike functions with respect to symmetric points in $\mathcal{U}$. Also $S_{\lambda, \mu}^{0}(\alpha, 0)=S^{*}(\alpha)$ and $T_{\lambda, \mu}^{0}(\alpha, 0)=C(\alpha)$ which are, respectively, the familiar classes of starlike functions of order $\alpha(0 \leq \alpha<1)$ and convex functions of order $\alpha(0 \leq \alpha<1)$. Incidentally the class of uniformly starlike functions introduced by Goodman [12] as follows

$$
U S T=\left\{f(z) \in \mathcal{A}: \Re\left(\frac{(z-\zeta) f^{\prime}(z)}{f(z)-f(\zeta z)}\right)>0\right\}, \quad(z, \zeta) \in \mathcal{U} \times \mathcal{U} .
$$

Ronning [13] showed the following important result.

Remark 1.1. $f(z) \in U S T$ if and only if for every $z \in \mathcal{U},|t|=1$

$$
\Re\left(\frac{(1-t) z f^{\prime}(z)}{f(z)-f(t z)}\right)>0 .
$$

Now we will give some results for functions belonging to the classes $S_{0, \lambda, \mu}^{n}(\alpha, t)$ and $T_{0, \lambda, \mu}^{n}(\alpha, t)$. 
2. $S_{0, \lambda, \mu}^{n}(\alpha, t)$ AND $T_{0, \lambda, \mu}^{n}(\alpha, t)$

Theorem 2.1. If $f(z) \in \mathcal{A}$ satisfies

$$
\begin{aligned}
& \sum_{k=2}^{\infty} A_{k}^{n}\left\{\left|k-u_{k}\right|+(1-\alpha)\left|u_{k}\right|\right\}\left|a_{k}\right| \leq 1-\alpha, \\
& u_{k}=1+t+t^{2}+\cdots+t^{k-1}, t(|t| \leq 1, t \neq 1)
\end{aligned}
$$

for some $\alpha(0 \leq \alpha<1)$ then $f(z) \in S_{\lambda, \mu}^{n}(\alpha, t)$, where

$$
A_{k}^{n}=[1+(\lambda \mu k+\lambda-\mu)(k-1)]^{n} .
$$

Proof. To prove Theorem 2.1, we show that if $f(z)$ satisfies $(2.1)$ then

$$
\left|\frac{(1-t) z\left(D_{\lambda, \mu}^{n} f(z)\right)^{\prime}}{D_{\lambda, \mu}^{n} f(z)-D_{\lambda, \mu}^{n} f(t z)}-1\right|<1-\alpha .
$$

Evidently, since

$$
\frac{(1-t) z\left(D_{\lambda, \mu}^{n} f(z)\right)^{\prime}}{D_{\lambda, \mu}^{n} f(z)-D_{\lambda, \mu}^{n} f(t z)}-1=\frac{z+\sum_{k=2}^{\infty} k A_{k}^{n} a_{k} z^{k}}{z+\sum_{k=2}^{\infty} k A_{k}^{n} u_{k} a_{k} z^{k}}-1=\frac{\sum_{k=2}^{\infty}\left(k-u_{k}\right) A_{k}^{n} a_{k} z^{k-1}}{1+\sum_{k=2}^{\infty} A_{k}^{n} u_{k} a_{k} z^{k-1}}
$$

we see that

$$
\left|\frac{(1-t) z\left(D_{\lambda, \mu}^{n} f(z)\right)^{\prime}}{D_{\lambda, \mu}^{n} f(z)-D_{\lambda, \mu}^{n} f(t z)}-1\right| \leq \frac{\sum_{k=2}^{\infty} A_{k}^{n}\left|k-u_{k}\right|\left|a_{k}\right|}{1-\sum_{k=2}^{\infty} A_{k}^{n}\left|u_{k}\right|\left|a_{k}\right|} .
$$

Therefore, if $f(z)$ satisfies (2.1), then we have

$$
\left|\frac{(1-t) z\left(D_{\lambda, \mu}^{n} f(z)\right)^{\prime}}{D_{\lambda, \mu}^{n} f(z)-D_{\lambda, \mu}^{n} f(t z)}-1\right|<1-\alpha .
$$

This completes the proof of Theorem 2.1.

Theorem 2.2. If $f(z) \in \mathcal{A}$ satisfies

$\sum_{k=2}^{\infty} k A_{k}^{n}\left\{\left|k-u_{k}\right|+(1-\alpha)\left|u_{k}\right|\right\}\left|a_{k}\right| \leq 1-\alpha, \quad u_{k}=1+t+t^{2}+\cdots+t^{k-1}$

for some $\alpha(0 \leq \alpha<1)$ then $f(z) \in T_{\lambda, \mu}^{n}(\alpha, t)$, where

$$
A_{k}^{n}=[1+(\lambda \mu k+\lambda-\mu)(k-1)]^{n} .
$$


Proof. Noting that $f \in T_{\lambda, \mu}^{n}(\alpha, t)$ if and only if $z f^{\prime} \in S_{\lambda, \mu}^{n}(\alpha, t)$, we can prove Theorem 2.2.

We now define

$$
S_{0, \lambda, \mu}^{n}(\alpha, t)=\{f \in \mathcal{A}: f \text { satisfies }(2.1)\}
$$

and

$$
T_{0, \lambda, \mu}^{n}(\alpha, t)=\{f \in \mathcal{A}: f \text { satisfies }(2.2)\} .
$$

In view of the above theorems, we see :

Example 2.1. Let us consider a function $f(z)$ given by

$$
\begin{array}{r}
f(z)=z+(1-\alpha)\left(\frac{\eta \delta_{2}}{2 A_{2}^{n}(2-\alpha)} z^{2}+\frac{(1-\eta) \delta_{3}}{A_{3}^{n}(7-3 \alpha)} z^{3}\right), \\
0 \leq \eta \leq 1, \quad\left|\delta_{2}\right|=\left|\delta_{3}\right|=1 .
\end{array}
$$

Then for any $t(|t| \leq 1, t \neq 1), f(z) \in S_{0, \lambda, \mu}^{n}(\alpha, t) \subset S_{\lambda, \mu}^{n}(\alpha, t)$.

Example 2.2. Let us consider a function $f(z)$ given by

$$
\begin{aligned}
f(z)=z+(1-\alpha)\left(\frac{\eta \delta_{2}}{4 A_{2}^{n}(2-\alpha)} z^{2}+\frac{(1-\eta) \delta_{3}}{3 A_{3}^{n}(7-3 \alpha)} z^{3}\right), \\
0 \leq \eta \leq 1, \quad\left|\delta_{2}\right|=\left|\delta_{3}\right|=1 .
\end{aligned}
$$

Then for any $t(|t| \leq 1, t \neq 1), f(z) \in T_{0, \lambda, \mu}^{n}(\alpha, t) \subset T_{\lambda, \mu}^{n}(\alpha, t)$.

Remark 2.3. If we take $n=0, t=-1$ in Theorems 2.1 and 2.2 then we get the results given by Cho et al. [6].

\section{Coefficient inequalities}

Applying Caratheodory function $p(z)$ defined by

$$
p(z)=1+\sum_{k=1}^{\infty} p_{k} z^{k}
$$

in $\mathcal{U}$, we discuss the coefficient inequalities for the functions $f$ in the subclasses $S_{\lambda, \mu}^{n}(\alpha, t)$ and $T_{\lambda, \mu}^{n}(\alpha, t)$. 
Theorem 3.1. If $f(z) \in S_{\lambda, \mu}^{n}(\alpha, t)$, then

$$
\begin{aligned}
\left|a_{k}\right| \leq \frac{\beta}{A_{k}^{n}\left|v_{k}\right|}\{1 & +\beta \sum_{j=2}^{k-1} \frac{\left|u_{j}\right|}{\left|v_{j}\right|}+\beta^{2} \sum_{j_{2}>j_{1}}^{k-1} \sum_{j_{1}=2}^{k-2} \frac{\left|u_{j_{1}} u_{j_{2}}\right|}{\left|v_{j_{1}} v_{j_{2}}\right|} \\
& \left.+\beta^{3} \sum_{j_{3}>j_{2}}^{k-1} \sum_{j_{2}>j_{1}}^{k-2} \sum_{j_{1}=2}^{k-3} \frac{\left|u_{j_{1}} u_{j_{2}} u_{j_{3}}\right|}{\left|v_{j_{1}} v_{j_{2}} v_{j_{3}}\right|}+\cdots+\beta^{k-2} \prod_{j=2}^{k-1} \frac{\left|u_{j}\right|}{\left|v_{j}\right|}\right\},
\end{aligned}
$$

where

$$
\beta=2(1-\alpha), v_{k}=k-u_{k} .
$$

Proof. We define the function $p(z)$ by

$$
p(z)=\frac{1}{1-\alpha}\left(\frac{(1-t) z\left(D_{\lambda, \mu}^{n} f(z)\right)^{\prime}}{D_{\lambda, \mu}^{n} f(z)-D_{\lambda, \mu}^{n} f(t z)}-\alpha\right)=1+\sum_{k=1}^{\infty} p_{k} z^{k}
$$

for $f(z) \in S_{\lambda, \mu}^{n}(\alpha, t)$. Then $p(z)$ is a Caratheodory function and satisfies

$$
\left|p_{k}\right| \leq 2(k \geq 1)
$$

Since

$$
(1-t) z\left(D_{\lambda, \mu}^{n} f(z)\right)^{\prime}=\left[D_{\lambda, \mu}^{n} f(z)-D_{\lambda, \mu}^{n} f(t z)\right][\alpha+(1-\alpha) p(z)],
$$

we have

$$
z+\sum_{k=2}^{\infty} k A_{k}^{n} a_{k} z^{k}=\left(z+\sum_{k=2}^{\infty} k A_{k}^{n} u_{k} a_{k} z^{k}\right)\left(1+(1-\alpha) \sum_{k=1}^{\infty} p_{k} z^{k}\right)
$$

where

$$
u_{k}=1+t+t^{2}+\cdots+t^{k-1}
$$

So we get

$$
\begin{aligned}
a_{k}=\frac{1-\alpha}{A_{k}^{n}\left(k-u_{k}\right)}\left(p_{1} A_{k-1}^{n} u_{k-1} a_{k-1}+p_{2} A_{k-2}^{n} u_{k-2} a_{k-2}+\ldots\right. \\
\left.+p_{k-2} A_{2}^{n} u_{2} a_{2}+p_{k-1}\right) .
\end{aligned}
$$

From Eq. (3.5), we easily have that

$$
\begin{gathered}
\left|a_{2}\right|=\left|\frac{(1-\alpha)}{A_{2}^{n}\left(2-u_{2}\right)} p_{1}\right| \leq \frac{2(1-\alpha)}{A_{2}^{n}\left|2-u_{2}\right|} \\
\left|a_{3}\right| \leq \frac{2(1-\alpha)}{A_{3}^{n}\left|3-u_{3}\right|}\left(A_{2}^{n}\left|u_{2} a_{2}\right|+1\right) \leq \frac{2(1-\alpha)}{A_{3}^{n}\left|3-u_{3}\right|}\left(1+2(1-\alpha) \frac{\left|u_{2}\right|}{\left|2-u_{2}\right|}\right)
\end{gathered}
$$


and

$$
\begin{array}{r}
\left|a_{4}\right| \leq \frac{2(1-\alpha)}{A_{4}^{n}\left|4-u_{4}\right|}\left\{1+2(1-\alpha)\left(\frac{\left|u_{2}\right|}{\left|2-u_{2}\right|}+\frac{\left|u_{3}\right|}{\left|3-u_{3}\right|}\right)\right. \\
\left.+2^{2}(1-\alpha)^{2} \frac{\left|u_{2} u_{3}\right|}{\left|2-u_{2}\right|\left|3-u_{3}\right|}\right\} .
\end{array}
$$

Thus, using the mathematical induction, we obtain the inequality (3.2).

Remark 3.2. If we write $\alpha=t=n=0$ in Theorem 3.1 then we have well known the result

$$
f \in S^{*} \Longrightarrow\left|a_{k}\right| \leq k,
$$

where $S^{*}$ is usual the class of starlike functions.

Remark 3.3. If we take $\alpha=\frac{1}{2}, t=0, n=1, \lambda=1, \mu=0$ in Theorem 3.1 then we obtain

$$
\left|a_{k}\right| \leq \frac{1}{k}
$$

Remark 3.4. If we take $\alpha=0, t=-1, n=1$, in Theorem 3.1 then we have

$$
\left|a_{k}\right| \leq \frac{1}{A_{k}}
$$

where $A_{k}=1+(\lambda \mu k+\lambda-\mu)(k-1)$ and $\lambda \geqslant \mu \geqslant 0$.

Remark 3.5. If we put $\lambda=\mu=1$ in Remark 3.4 then we have the following

$$
\left|a_{k}\right| \leq \frac{1}{k^{2}-k+1} \text {. }
$$

Remark 3.6. Equalities in Theorem 3.1 are attended for $f(z)$ given by

$$
\frac{(1-t) z\left(D_{\lambda, \mu}^{n} f(z)\right)^{\prime}}{D_{\lambda, \mu}^{n} f(z)-D_{\lambda, \mu}^{n} f(t z)}=\frac{1+(1-2 \alpha) z}{1-z} .
$$

Theorem 3.7. If $f(z) \in T_{\lambda, \mu}^{n}(\alpha, t)$, then

$$
\begin{aligned}
\left|a_{k}\right| \leq \frac{\beta}{k A_{k}^{n}\left|v_{k}\right|}\left\{1+\beta \sum_{j=2}^{k-1} \frac{\left|u_{j}\right|}{\left|v_{j}\right|}+\beta^{2} \sum_{j_{2}>j_{1}}^{k-1} \sum_{j_{1}=2}^{k-2} \frac{\left|u_{j_{1}} u_{j_{2}}\right|}{\left|v_{j_{1}} v_{j_{2}}\right|}\right. \\
\left.+\beta^{3} \sum_{j_{3}>j_{2}}^{k-1} \sum_{j_{2}>j_{1}}^{k-2} \sum_{j_{1}=2}^{k-3} \frac{\left|u_{j_{1}} u_{j_{2}} u_{j_{3}}\right|}{\left|v_{j_{1}} v_{j_{2}} v_{j_{3}}\right|}+\cdots+\beta^{k-2} \prod_{j=2}^{k-1} \frac{\left|u_{j}\right|}{\left|v_{j}\right|}\right\}
\end{aligned}
$$

where

$$
\beta=2(1-\alpha), v_{k}=k-u_{k}
$$




\section{Distortion inequalities}

For the functions $f(z)$ in the classes $S_{0, \lambda, \mu}^{n}(\alpha, t)$ and $T_{0, \lambda, \mu}^{n}(\alpha, t)$, we derive Theorem 4.1. If $f(z) \in S_{0, \lambda, \mu}^{n}(\alpha, t)$, then

$$
|z|-\sum_{k=2}^{j}\left|a_{k}\right||z|^{k}-B_{j}|z|^{j+1} \leq|f(z)| \leq|z|+\sum_{k=2}^{j}\left|a_{k}\right||z|^{k}+B_{j}|z|^{j+1}
$$

where

$$
B_{j}=\frac{1-\alpha-\sum_{k=2}^{j} A_{k}^{n}\left\{\left|k-u_{k}\right|+(1-\alpha)\left|u_{k}\right|\right\}\left|a_{k}\right|}{\left(j+1-\alpha\left|u_{j+1}\right|\right) A_{j+1}^{n}} \quad(j \geq 2) .
$$

Proof. From the inequality (2.1) we know that

$$
\begin{aligned}
\sum_{k=j+1}^{\infty} A_{k}^{n}\left\{\left|k-u_{k}\right|+(1-\alpha)\left|u_{k}\right|\right\} & \left|a_{k}\right| \leq 1-\alpha \\
& -\sum_{k=2}^{j} A_{k}^{n}\left\{\left|k-u_{k}\right|+(1-\alpha)\left|u_{k}\right|\right\}\left|a_{k}\right| .
\end{aligned}
$$

On the other hand

$$
\left\{\left|k-u_{k}\right|+(1-\alpha)\left|u_{k}\right|\right\} \geq k-\alpha\left|u_{k}\right|,
$$

and $k-\alpha\left|u_{k}\right|$ is monotonically increasing with respect to $k$. Thus we deduce $\left(j+1-\alpha\left|u_{j+1}\right|\right) A_{j+1}^{n} \sum_{k=j+1}^{\infty}\left|a_{k}\right| \leq 1-\alpha-\sum_{k=2}^{j} A_{k}^{n}\left\{\left|k-u_{k}\right|+(1-\alpha)\left|u_{k}\right|\right\}\left|a_{k}\right|$, which implies that

$$
\sum_{k=j+1}^{\infty}\left|a_{k}\right| \leq B_{j}
$$

Therefore we have the following

$$
|f(z)| \leq|z|+\sum_{k=2}^{j}\left|a_{k}\right||z|^{k}+B_{j}|z|^{j+1}
$$

and

$$
|f(z)| \geq|z|-\sum_{k=2}^{j}\left|a_{k}\right||z|^{k}-B_{j}|z|^{j+1} .
$$

This completes the proof of theorem.

Similarly we have 
Theorem 4.2. If $f(z) \in T_{0, \lambda, \mu}^{n}(\alpha, t)$, then

$$
|z|-\sum_{k=2}^{j}\left|a_{k}\right||z|^{k}-C_{j}|z|^{j+1} \leq|f(z)| \leq|z|+\sum_{k=2}^{j}\left|a_{k}\right||z|^{k}+C_{j}|z|^{j+1}
$$

and

$$
1-\sum_{k=2}^{j} k\left|a_{k}\right||z|^{k-1}-D_{j}|z|^{j} \leq\left|f^{\prime}(z)\right| \leq 1+\sum_{k=2}^{j} k\left|a_{k}\right||z|^{k-1}+D_{j}|z|^{j}
$$

where

$$
C_{j}=\frac{1-\alpha-\sum_{k=2}^{j} k A_{k}^{n}\left\{\left|k-u_{k}\right|+(1-\alpha)\left|u_{k}\right|\right\}\left|a_{k}\right|}{(j+1)\left\{j+1-\alpha\left|u_{j+1}\right|\right\} A_{j+1}^{n}} \quad(j \geq 2)
$$

and

$$
D_{j}=\frac{1-\alpha-\sum_{k=2}^{j} k A_{k}^{n}\left\{\left|k-u_{k}\right|+(1-\alpha)\left|u_{k}\right|\right\}\left|a_{k}\right|}{\left\{j+1-\alpha\left|u_{j+1}\right|\right\} A_{j+1}^{n}} \quad(j \geq 2) .
$$

Remark 4.3. If we choice $n=0, t=-1, j=2$ in Theorems 4.1 and 4.2 , then we get the results given by Cho et al. [6].

\section{Relation Between the Classes} have

By the definitions for the classes $S_{0, \lambda, \mu}^{n}(\alpha, t)$ and $T_{0, \lambda, \mu}^{n}(\alpha, t)$, evidently we

$$
S_{0, \lambda, \mu}^{n}(\alpha, t) \subset S_{0, \lambda, \mu}^{n}(\beta, t) \quad(0 \leq \beta \leq \alpha<1)
$$

and

$$
T_{0, \lambda, \mu}^{n}(\alpha, t) \subset T_{0, \lambda, \mu}^{n}(\beta, t) \quad(0 \leq \beta \leq \alpha<1) .
$$

Let us consider a relation between $S_{0, \lambda, \mu}^{n}(\alpha, t)$ and $T_{0, \lambda, \mu}^{n}(\alpha, t)$.

Theorem 5.1. If $f(z) \in T_{0, \lambda, \mu}^{n}(\alpha, t)$, then $f(z) \in S_{0, \lambda, \mu}^{n}\left(\frac{1+\alpha}{2}, t\right)$.

Proof. Let $f(z) \in T_{0, \lambda, \mu}^{n}(\alpha, t)$. Then if $f(z)$ satisfies

$$
\frac{\left|k-u_{k}\right|+(1-\beta)\left|u_{k}\right|}{1-\beta} \leq k \frac{\left|k-u_{k}\right|+(1-\alpha)\left|u_{k}\right|}{1-\alpha}
$$

for all $k \geq 2$, then we have that $f(z) \in S_{0, \lambda, \mu}^{n}(\beta, t)$. From (5.1), we have

$$
\beta \leq 1-\frac{(1-\alpha)\left|k-u_{k}\right|}{k\left|k-u_{k}\right|+(1-\alpha)(k-1)\left|u_{k}\right|} .
$$


Furthermore, since for all $k \geq 2$

$$
\frac{\left|k-u_{k}\right|}{k\left|k-u_{k}\right|+(1-\alpha)(k-1)\left|u_{k}\right|} \leq \frac{1}{k} \leq \frac{1}{2},
$$

we obtain

$$
f(z) \in S_{0, \lambda, \mu}^{n}\left(\frac{1+\alpha}{2}, t\right) .
$$

Remark 5.2. Taking $n=0$ in Theorems 2.1- 5.1, we immediately obtain the results due to Owa et al. [7].

Remark 5.3. If we put $n=0, t=-1$ in Theorems 3.1- 5.1, then we get the results given by Owa et al. [8].

\section{REFERENCES}

[1] D. Răducanu and H. Orhan, Subclasses of analytic functions defined by a generalized differential operator, Int. J. Math. Anal., 4 (1) (2010) 1-15.

[2] E. Deniz, H. Orhan, The Fekete Szegö problem for a generalized subclass of analytic functions, Kyungpook Math. J. 50 (2010) 37-47.

[3] F. M. Al-Oboudi, On univalent functions defined by a generalized Salagean operator, Int. J. Math. Sci., 27 (2004) 1429-1436.

[4] G. S. Sălăgean, Subclasses of univalent functions, Comp. Anal.-Proc. 5th Rom.Finnish Seminar, Bucharest 1981, Part 1, Lec. Notes Math., 1013 (1983) 362-372.

[5] K. Sakaguchi, On a certain univalent mapping, J. Math. Soc., Japan 11 (1959) 72-75.

[6] N. E. Cho, O. S. Kwon, S. Owa, Certain subclasses of Sakaguchi functions, SEA Bull. Math., 17 (1993) 121-126.

[7] S. Owa, T. Sekine, Rikuo Yamakawa, On Sakaguchi type functions, Appl. Math. Comp., 187 (2007) 356-361.

[8] S. Owa, T. Sekine, Rikuo Yamakawa, Notes on Sakaguchi functions, RIMS, Kokyuroku, 1414 (2005) 76-82.

[9] B. A. Frasin, Coefficient inequalities for certain classes of Sakaguchi type functions, Int. J. Nonlinear Sci., 10 (2) (2010), 206-211.

[10] Y. Polatoğlu, E. Yavuz, Multivalued Sakaguchi functions, Gen. Math., 15 (2-3) (2007), $132-140$.

[11] B. A. Frasin, M. Darus, Subordination results on subclasses concerning Sakaguchi functions, J. Inequal. Appl., 2009, Article ID 574014.

[12] A. W. Goodman, On uniformly starlike functions, J. Math. Anal. Appl., 155 (1991), $364-370$.

[13] F. Ronning, On uniform starlikeness and related properties of univalent functions, Complex Variables, Theory Appl., 24 (1994) 233-239. 
E-mails: orhanhalit607@gmail.com mcaglar25@gmail.com

Nihat Yagmur

Department of Mathematics

Faculty of Science and Art

Erzincan University

24100, Erzincan, Turkey

E-mail: nhtyagmur@gmail.com 